\title{
MedienPädagogik
}

Zeitschrift für Theorie und Praxis der Medienbildung

www.medienpaed.com

\section{Grosse Erwartungen: Die Rolle von Einstellungen bei der Nutzung und Verbreitung von Open Educational Resources}

Daniel Otto

\begin{abstract}
Zusammenfassung
Open Educational Resources (OER) sind ein prominenter Gegenstand der Bildungsdiskussion. OER wird das Potenzial zugeschrieben, die Entwicklung partizipativer und kollaborativer Lernszenarien zu ermöglichen und auf einer grundlegenderen Ebene offene Bildung und Bildungsgerechtigkeit zu fördern und zu unterstützen. Dennoch ist die Verbreitung von OER in der Bildung bisher eher episodisch denn systematisch. Der Beitrag versucht auf Basis der Einstellungsforschung eine empirische Annäherung an die Rolle von Einstellung bei der Nutzung und Verbreitung von OER. Auf Grundlage eines umfragebasierten explorativen Ansatzes wurde versucht, die determinierenden Einstellungskomponenten bildungsbereichsübergreifend abzubilden. Die Ergebnisse zeigen, dass Einstellungen nicht das zentrale Hindernis für die Nutzung von OER sind, sondern die mangelnde Verbreitung. Bezüglich der Verbreitung lässt sich konstatieren, dass sowohl Intention als auch Verhalten nur innerhalb einer breiteren OER-Gemeinschaft existieren, die allerdings starke Emotionen und Überzeugungen mit den Kernideen und Mehrwerten von OER verbindet. Diesem Argument folgend ist der häufig geforderte Abbau struktureller Barrieren ein notwendiger, aber keineswegs hinreichender Grund, um die Nutzung und Verbreitung von OER zu erhöhen. Es ist zu empfehlen, OER nicht als ein normatives Element einer Openness-Bewegung zu konzipieren, sondern als eine (Teil-)Antwort auf die Herausforderung des Lehrens und Lernens in der digitalen Welt zu begreifen und zu fördern.
\end{abstract}

Great expectations: the role of attitudes in the use and distribution of Open Educational Resources

\begin{abstract}
Open Educational Resources (OER) are a prominent topic of the educational discourse. $O E R$ is understood as facilitating participatory and collaborative learning scenarios and to promote and support open education and educational justice. Nevertheless, the adoption of OER in education has so far been episodic rather than systematic. Based on the concept of attitudes, this paper seeks an empirical approach to the role of attitudes in the adoption of OER. Built on a survey-based explorative approach, an attempt was made to map the determining components of attitudes across educational sectors. The results
\end{abstract}


demonstrate that attitudes are not the central obstacle to the use of OER. However, it can be stated that both intention and behaviour exist only within a broader OER community, which associates strong emotions and beliefs with the core ideas and added values of OER. Following this argument, the frequently demanded dismantling of structural barriers is a necessary but by no means sufficient cause to increase the adoption of OER. In this respect, it is advisable to perceive OER not as a normative element of an openness movement, but as a (partial) answer to a broader challenge of teaching and learning in the digital world.

\section{Einleitung}

Nicht erst seit dem Bericht des UNESCO Forum on the Impact of Open Courseware for Higher Education in Developing Countries im Jahr 2002 sind Open Educational Resources (OER), im Deutschen meist als Offene Bildungsmaterialien bezeichnet, ein prominenter Gegenstand der Bildungsdiskussion (UNESCO 2002).

Die Idee von OER reiht sich ein in eine grundlegendere Forderung nach einer Ausrichtung der Bildung und Wissenschaft hin zur Offenheit. Für die Forderung nach der Offenheit der Wissenschaft (Open Science) wird wiederholt die Metapher eines Open Science Schirms bemüht, unter dessen Schutz sich neben OER weitere Konzepte wie Open Access, Open Data und Open Source begeben (Fecher und Friesike 2014). Bildungspolitisch wird OER unter eine grundlegendere Forderung nach Partizipation und Bildungsgerechtigkeit im digitalen Zeitalter subsumiert, die als Open Education bezeichnet wird (Deimann 2018). Letzteres Ansinnen wird auch durch die jüngst von der UNESCO veröffentlichten Richtlinien zur Erarbeitung von OER-Policies deutlich, die besonders den Beitrag von OER zu der Agenda 2030 und dessen Nachhaltigkeitsziel (SDGs) 4 (Hochwertige Bildung) betonen (UNESCO 2019).

Auch in der bildungswissenschaftlichen Diskussion erfährt die Auseinandersetzung mit Konzepten der Offenheit zunehmende Aufmerksamkeit. So bestätigen systematische Analysen der internationalen Forschungsliteratur das inkrementelle Interesse an Openness und Open Education, insbesondere an OER (Bozkurt, Koseoglu, und Singh 2019). Obwohl die grundsätzlichen Bestrebungen hin zu Offenheit überwiegend positiv konnotiert sind, wird von Kritikern angeführt, dass "'Openness' has become a highly charged and politicised term, a movement operating in many areas outside of education» (Bayne, Knox, und Ross 2015, 247). Autoren wie Kerres (2019) hinterfragen daher vor allem die Interpretation einer reziproken Beziehung der verschieden Konzepte von Offenheit, die häufig ein kaskadisches Verständnis nahelegen. Dies manifestiert sich im Fall von OER darin, dass diese stets in einem breiteren Open Education Kontext betrachtet werden (Deimann 2018). Das OER in dieser Kaskade hin zu Open Education eine basale Funktion einnimmt, zeigen besonders die durch OER ermöglichten Folgekonzepte (OER-enabled) einer OER-enabled 
Pedagogy (OP) (Wiley und Hilton 2018) sowie von OER-enabled Open Educational Practices (OEP) (OPAL 2011). OP erweitert die Freiheiten von OER um ein pädagogisches Konzept, das Lernende als aktive Gestaltende im Lernprozess versteht. Von ihnen erstellte Artefakte besitzen über den eigentlichen Lernprozess hinaus einen Wert und sollen daher unter einer offenen Lizenz verbreitet und weiter genutzt werden. Weniger eindeutig ist bislang die Definition und Reichweite von OEP. So zeigt eine Analyse von Bellinger und Mayrberger (2019), dass in der Literatur einerseits ein sehr weites Verständnis von OEP postuliert wird, dass auch institutionelle Perspektiven und andere Konzepte der offenen Bildung einbezieht. Andererseits existiert parallel ein enges Verständnis von OEP, welches auf die Verwendung von OER in offenen oder geschlossenen Lernszenarien beschränkt bleibt.

Als Ergebnis lässt sich festhalten, dass sich OER in einem Dualismus zwischen einer normativen Forderung nach offenerer Bildung und Bildungsgerechtigkeit auf der einen und auf der Suche nach einer adäquaten (medien-)pädagogischen Begründung für OER auf der anderen Seite befindet. Speziell die normative Wertebegründung für OER kulminierte in der Literatur teilweise in einer Selbstzuschreibung, Teil einer Openness-Bewegung (Openness Movements) zu sein (siehe Gourlay 2015; Peters und Peter Roberts 2011; Peters 2008), die sich als Gegennarrativ zu Geschlossenheit positioniert.

Rekurrierend auf die Emergenz von OER im Bildungsdiskurs und der Vielfalt der Deutungsperspektiven scheint es für eine fundierte medienpädagogische Betrachtung hilfreich, sich den gegenwärtigen Status von OER im deutschsprachigen Raum genauer zu erschliessen.

Konträr zur ambivalenten Deutung scheint definitorisch weitestgehend Einigkeit darüber zu bestehen, was unter OER zu verstehen ist. Ein implizit und explizit vorherrschendes Verständnis definiert OER als

«any educational resources (including curriculum maps, course materials, textbooks, streaming videos, multimedia applications, podcasts, and any other materials that have been designed for use in teaching and learning) that are openly available for use by educators and students, without an accompanying need to pay royalties or licence fees» (Kanwar und Uvalić-Trumbić 2011, 4)

Die Offenheit dieser Bildungsmaterialien soll Lehrenden und Lernenden die so genannten «5 V-Freiheiten für Offenheit» ermöglichen, also das Verwahren/Vervielfältigen, Verwenden, Verarbeiten, Vermischen und Verbreiten von Bildungsmaterialien (Wiley und Hilton 2018). Unter anderem auf dieser Grundlage wird OER das Potenzial zugeschrieben, die Entwicklung partizipativer und kollaborativer Lernszenarien zu ermöglichen. 
Deutschland kann bezüglich OER im internationalen Vergleich eher als Nachzügler verstanden werden (Orr, Neumann, und Muuß-Merholz 2017). So waren bei den ersten Bemühungen, OER auf internationaler Ebene durch die Initiativen von UNESCO, OECD und anderen Akteuren wie dem OpenCourseWare Consortium (OCWC) zu etablieren, mit wenigen Ausnahmen, keine deutschen Vertreterinnen oder Vertreter präsent (Deimann, Neumann, und Muuß-Merholz 2015). Ein im Jahr 2017 von Orr, Neumann und Muuß-Merholz (2017) erstellter Bericht bietet eine Übersicht über die OER-bezogenen Entwicklung in Deutschland und beschreibt die wesentlichen Herausforderungen. Obwohl in dem Bericht 166 Organisationen identifiziert wurden, die in OER-bezogene Aktivitäten involviert sind, wird Deutschland vor allem hinsichtlich der Nutzung und der Verbreitung als Nachzügler eingeordnet.

Erst durch die jüngste Förderlinie des Bundesministeriums für Bildung und Forschung (BMBF), OERinfo (2017/2018), ist der Versuch unternommen worden, das Thema aus seinem Nischendasein zu befreien und die Nutzung und Verbreitung von OER deutschlandweit zu erhöhen (Surmann und Echterhoff 2018). Die Förderung umfasste einerseits die Bildung eines zentralen Informationsportals für die Allokation und Distribution von Informationen rund um OER (OERinfo), die vom Leibniz-Institut für Bildungsforschung und Bildungsinformation (DIPF) verwaltet wird sowie andererseits die Förderung von 24 weiteren OER-Projekten, in denen bildungsbereichsübergreifend Massnahmen zur Qualifizierung von Akteurinnen und Akteuren für die Nutzung und Weiterqualifizierung von OER durchgeführt wurden (Mayrberger 2018).

In der Folge haben viele Bundesländer und Hochschulen begonnen, auf technischer Ebene OER-Repositorien einzurichten. Diese dienen nicht nur dazu, Lehrenden und Lernenden die Möglichkeit zu bieten, OER abzurufen, sondern auch eigene Inhalte bereitzustellen. In jüngsten Forschungsprojekten wird versucht, diese Repositorien auf prototypischer Ebene durch den Austausch von Metadaten zu vernetzen (Kerres u.a. 2019).

Trotz all dieser Bestrebungen bestätigt der jüngste Bericht der UNESCO die rudimentäre Verbreitung und Nutzung von OER in Deutschland:

«In Germany, OER adoption is also low, particularly outside the community of German OER experts in all sectors of education and training» (UNESCO IITE 2019, 27).

Es lässt sich beobachten, dass während die Anzahl von Repositorien und die Quantität von Inhalten langsam aber stetig ansteigt, sich besonders Praktiken im Umgang mit OER aber eher episodisch denn systematisch ereignen (Otto 2019) und bislang häufig auf «Selbstversuche» beschränkt bleiben (siehe Lechtenbörger 2019). Dies bestätigen ebenso die jüngsten Umfragen des Monitors Digitale Bildung für die Schule (Schmid, Goertz, und Behrens 2017) und die Hochschule, die attestieren, «beim Umgang mit offenen Lernmaterialien (OER) herrscht unter den Lehrenden Zurückhaltung» (Schmid u.a. 2017, 7). 
Eine empirische Annäherung an den Status der Verbreitung (Kenntnis von) und Nutzung (Praktiken mit) von OER ist daher aus praxeologischer Sicht interessant. Dies wird als Möglichkeit verstanden, soziale Praktiken zu identifizieren und deren Eigenlogik zu analysieren (Breidenstein 2009). Da allerdings bislang soziale Praktiken hinsichtlich OER für den deutschen Fall nur bedingt existieren oder bislang unbekannt sind, fokussiert der vorliegende Beitrag nicht die Analyse existierender OER-Praktiken, sondern untersucht die determinierenden kognitiven (Wissen und Überzeugungen) und affektiven (Gefühle und Emotionen) Einflussfaktoren in Bezug auf das (selbsteingeschätze) Verhalten. Die erwartete Relevanz der Faktoren von Überzeugungen und Emotionen geschieht auch im Rekurs auf den eingangs geschilderten Dualismus von (medien-)pädagogischer (inhaltlicher) und normativer (wertebasierter) Begründung, wobei beides nicht immer eindeutig voneinander zu trennen ist. Die normative Aufladung der Debatte über OER und nicht zuletzt die Begriffsprägung einer Openness-Bewegung lässt eine hohe Bedeutung von Überzeugungen und Emotionen zumindest vermuten. Allgemein erfährt die Erforschung des Einfluss von Emotionen und Überzeugungen bei der Entstehung und Perpetuierung sozialer und politischer Bewegungen zunehmende Aufmerksamkeit und wird in der Forschungsliteratur kaum mehr bestritten (Flam und King 2005; Jasper 2011). Bedeutung und Wirkmächtigkeit dieser Faktoren scheinen daher auch für den Fall der Verbreitung und Nutzung von OER in Deutschland interessant.

Unter Bezugnahme auf die Befunde der Einstellungsforschung ist daher für die folgenden Ausführungen der (potentielle) Einfluss von kognitiven und affektiven Faktoren für soziale Praktiken als axiomatisch zu verstehen (von Scheve 2011). Ceteres paribus, gilt im Umgang mit Medien und damit ebenfalls OER, der Einfluss dieser Faktoren auf das menschliche Handeln als evident (Stark, Park, und Brünken 2018). Die starke Ausprägung von Überzeugungen und Emotionen fungiert demnach bis zu einem gewissen Ausmass als Prädiktor für soziale Praktiken (Allen, Machleit, und Kleine 1992).

Zusammenfassend und basierend auf den geschilderten Annahmen versucht der Beitrag sich der Frage nach der Rolle von Überzeugungen und Emotionen bei der Nutzung und Verbreitung von OER in Deutschland empirisch anzunähern. In einem ersten Schritt wird versucht, Überzeugungen und Emotionen als integralen Bestandteil eines breiteren Konzeptes der Einstellungsforschung zu konzeptionieren. Auf theoretischer Ebene greift der Beitrag dafür auf das Konzept der attitudes nach Rosenberg und Hovland (1960) zurück. Dieses Konzept wurde in Form eines Fragebogens für eine empirische Untersuchung operationalisiert und bildungsbereichsübergreifend distribuiert. Die Ergebnisse sowie deren Interpretation werden in den anschliessenden Kapiteln beschrieben. Eine Zusammenfassung und ein Ausblick für die zukünftigen Perspektiven von OER erfolgt im abschliessenden Kapitel. 
Eine (konzeptionelle) Annäherung an OER und die Rolle von Einstellungen

Obgleich die Anzahl wissenschaftlicher Veröffentlichungen über OER inkrementell ansteigt - wobei hier in erster Linie die USA und Grossbritannien dominant sind (Bozkurt, Koseoglu, und Singh 2019) - ist die empirische Forschung in Deutschland noch in den Anfängen (Deimann 2019). Während in Deutschland bisher generell wenige, veröffentlichte, wissenschaftliche Studien vorliegen, wird auf internationaler Ebene empirisch stark auf die strukturellen Gründe für die mangelnde Nutzung von OER fokussiert (Richter und Ehlers 2012; Percy und Van Belle 2012; Guo u.a. 2015). Als prävalente Faktoren wurden primär Zeitmangel, Rechtsunsicherheit sowie institutionelle Barrieren identifiziert (Bozkurt, Koseoglu, und Singh 2019). Bislang wenig adressiert wird dagegen eine akteurszentrierte Perspektive (Agency), die den strukturalistischen Ansätzen (Structure) gegenübergestellt werden kann. Konträr zu strukturdeterministischen Ansätzen und ihrem systemischen Holismus argumentieren akteurszentrierte Perspektiven auf Basis des methodologischen Individualismus, dass die Beschaffenheit der Struktur durch die Art des Handelns determiniert wird (Weede 1992).

Im vorliegenden Beitrag wird für die Bedeutung und den Einfluss von Überzeugungen und Emotionen aus einer akteurszentrierten Perspektive argumentiert. Dabei wird jedoch im Rückgriff auf Giddens (1979) Dualität der Struktur davon ausgegangen, dass keine der beiden Perspektiven eine exklusive Erklärung darstellt, sondern sich Akteur und Struktur wechselseitig konstituieren.

Für akteurszentrierte Perspektiven scheint es ratsam, sich auf psychologische Ansätze zu konzentrieren, da diese Einstellungen auf Basis bisheriger Erfahrungen untersuchen und darauf aufbauend auf zukünftiges Verhalten schliessen. Die Einstellungsforschung zählt dabei zu einem der am meisten angewandten Konzepte in der sozialwissenschaftlichen Forschung (Guyer und Fabrigar 2015). Das latente psychologische Konstrukt der Einstellungen wurde bereits früh als eines der unverzichtbarsten Konzepte der Sozialpsychologie charakterisiert (Allport 1935). Dies in erster Linie, weil das Konzept den Anspruch erhebt, bei Forschungsmethodischer Rigorosität eine Beziehung zwischen sozialer Einstellung und sozialem Handeln herstellen zu können (Meinefeld 1977). Unabhängig von dem Handlungsimperativ von Einstellungen lassen sich mittels der Einstellungsforschung die Werte und Überzeugungen von Personen bezüglich bestimmter Objekte oder Subjekte bestimmen.

In seiner basalen Ausrichtung beschreibt die Theorie der Einstellung positive oder negative Bewertungen einer Person gegenüber einer anderen Person, einem Ort, einer Sache oder einem Ereignis (Rosenberg und Hovland 1960). Eine in der Literatur weit verbreitete Definition konzipiert Einstellungen als «anything a person may hold in mind, ranging from the mundane to the abstract, including things, people, groups, and ideas» (Bohner und Dickel 2010, 392). Einstellungen können demnach als eine Art von Wissensstruktur beschrieben werden, die zum Zeitpunkt einer Beurteilung in 
den Erinnerungen präsent ist (Guyer und Fabrigar 2015). Obwohl Einstellungen damit als einfache Assoziationen zur Objektbewertung charakterisiert werden, können sie Teil grösserer Mengen von komplexen Wissensstrukturen sein.

Somit ist die klassische und prävalente Konzeptualisierung von Einstellungen, dass diese eine umfassende Evaluationen darstellen, auf die Personen aus dem Gedächtnis zugreifen können, wenn sie dazu aufgefordert werden.

Hinsichtlich der Morphologie einer Struktur von Einstellungen ist in der Literatur die dreiseitige Theorie beziehungsweise die Vorstellung von drei Einstellungskomponenten dominant (Leandre R. Fabrigar, Tara K. MacDonald 2005). Somit nimmt die dreiseitige Theorie an, dass Einstellungen aus drei Komponenten bestehen, die in nachfolgenden empirischen Untersuchungen nachgewiesen und voneinander unterschieden werden konnten.

Das daraus entstandene Komponentenmodell der Einstellung einer Person nach Rosenberg und Hovland (1960) postuliert eine Taxonomie, die eine kognitive (Wissen und Überzeugungen), eine affektive (Gefühle und Emotionen) sowie eine verhaltensbezogene (Handeln und Verhalten) Komponente umfassen. Dieses häufig verwendete Konzept von Einstellungen wird als das CAB- oder das ABC-Konzept bezeichnet (Rosenberg und Hovland 1960). Einstellungen können dabei sowohl explizit als auch implizit sein. Explizite Einstellungen werden bewusst wahrgenommen und können Verhalten eindeutig beeinflussen. Implizite Einstellungen sind unbewusst, haben aber dennoch Auswirkungen auf Verhaltensweisen.

Die Wechselbeziehung von Einstellungen und Verhalten wird in der Literatur weitestgehend anerkannt, was bedeutet, dass das Wissen über die Einstellungen einer Person zwar in keinem kausalen Verhältnis steht (Chaiklin 2011), das Verhalten aber in einem gewissen Ausmass vorhersagen kann (Veresova und Mala 2016). Das Handeln einer Person basiert demnach auf einer Absicht, die diesem spezifischen Verhalten vorausgeht. Besonders eine hohe Ausprägung von Gefühlen und Emotionen scheinen in diesem Prozess eine Schlüsselrolle zu spielen (Veresova und Mala 2016; Avey, Wernsing, und Luthans 2008; Allen, Machleit, und Kleine 1992).

\section{Methodik der explorativen Analyse}

Rekurrierend auf das ABC-Konzept der Einstellungen wurde dieses für eine empirische Untersuchung operationalisiert. Dabei wurde für jede der drei Komponenten von Einstellungen Indikatoren zur Bestimmung der Ausprägung bezüglich OER entwickelt (Tabelle 1). 


\begin{tabular}{|l|l|}
\hline Einstellungskomponente & Indikator zur Bestimmung der Ausprägung \\
\hline Kognitive Komponente & $\begin{array}{l}\text { Wissen und Überzeugungen über OER und deren erwarteten Poten- } \\
\text { ziale und Mehrwerte }\end{array}$ \\
\hline Affektive Komponente & $\begin{array}{l}\text { Gefühle und Emotionen, welche die mit OER assoziierten Werte } \\
\text { auslösen (teilen, kooperieren und austauschen) }\end{array}$ \\
\hline Handlungskomponente & $\begin{array}{l}\text { Erfahrungen mit OER oder die Absicht, sich mit diesen in der Praxis } \\
\text { zu beschäftigen }\end{array}$ \\
\hline
\end{tabular}

Tab. 1.: Operationalisierung der Einstellungskomponenten.

Als methodischer Ansatz für die empirische Messung von Einstellungen lassen sich zwei Grundtypen unterscheiden (Gawronski, Hofmann, und Wilbur 2006);

- direkte Messung (Likert-Skala und semantisches Differential)

- indirekte Messung (affektive Priming-Aufgaben und impliziter Assoziationstest)

Die intuitive Annahme, dass die direkte Messung bewusste Einstellungen widerspiegelt, während die indirekte Messung unbewusste Einstellungen ausdrückt, wird durch die Forschung nicht bestätigt (Gawronski, Hofmann, und Wilbur 2006). Somit muss die Form der Messung für jedes Forschungsdesign in erster Linie pragmatisch entschieden werden und ist durch die Randbedingungen des Forschungsgegenstands bestimmt. Für die vorliegende Untersuchung wurde die Entscheidung für eine Direktmessung also durch eben diese Randbedingungen (Forschungsobjekt) determiniert. Die Zielgruppe umfasste Lehrende und Lernende aus allen vier Bildungsbereichen in Deutschland, Schule, Hochschule, Weiterbildung und berufliche Bildung. Das übergeordnete Ziel der Messung war es, die Einstellung zu OER für möglichst viele Personen in allen Bildungsbereichen zu erheben. Daher wurde ein umfragebasierter Ansatz verwendet und ein Fragebogen entwickelt, um die Einstellung der Teilnehmenden gegenüber OER zu ermitteln. Der Fragebogen enthält vier Abschnitte mit insgesamt 14 Fragekategorien, von denen jeder eine Komponente der Einstellungen erfasst. Um eine möglichst hohe Rücklaufquote zu erzielen, wurde der Fragebogen so präzise und kurz wie möglich gestaltet, ohne die Reliabilität der Ergebnisse zu beeinträchtigen. Die Forschung betont, dass die Länge des Fragebogens und die Anwenderfreundlichkeit die Rücklaufquote signifikant beeinflussen (Dillman, Sinclair, und Clark 1993; Jepson u.a. 2005).

Neben soziodemographischen Fragen umfasste der Fragebogen insgesamt 23 Fragen, die entweder binär (ja oder nein) oder mittels einer Likert-Skala (1 völlige Ablehnung - 5 völlig Zustimmung) zu beantworten waren.

Die ersten fünf Fragen widmeten sich den Gefühlen und Emotionen der Befragungsteilnehmenden bezüglich Begriffen wie «Austausch» und «Zusammenarbeit» (affektive Komponente), welche Kerngedanken von OER repräsentieren. Im nächsten Abschnitt wurden die Teilnehmenden über ihr Wissen über das Konzept von OER 
befragt, gefolgt von sechs Aussagen, die Überzeugungen in Form des Mehrwertes von OER darstellen (kognitive Komponente), z.B. inwieweit die Teilnehmenden überzeugt sind, dass OER den Austausch von Wissen oder innovatives Lehren oder Lernen ermöglicht oder erleichtert. Der letzte Abschnitt sollte das tatsächliche oder intendierte Verhalten hinsichtlich OER (Verhaltenskomponente) offenlegen. Schliesslich wurden die Personen neben den Einstellungen auch zu den wichtigsten Hindernissen für die Nutzung von OER in der Praxis befragt.

Für den Fragebogen erfolgte ein erster Pretest $(n=8)$ und ein zweiter Pretest mit einer Testgruppe $(n=20)$. Dieses Verfahren wurde durchgeführt, um die Objektivität, Zuverlässigkeit und Validität der Fragen der Befragung zu gewährleisten, indem die Antworten des ersten Pretests mit Antworten des zweiten Pretests verglichen werden (Grimm 2010).

Um die Umfrage innerhalb der Zielgruppe zu distribuieren, wurde ein doppelter Ansatz gewählt. Die Umfrage wurde über zwei Hauptkanäle verteilt: Über die Kommunikationskanäle von OERinfo, einem zentralen Informationsportal für OER in Deutschland und seinen Transferpartnern für die vier Bildungsbereiche, Schule, Hochschule, Weiterbildung und berufliche Bildung. Es wurde dabei ein breiter Zugang gewählt, um auch Personen zu adressieren, die nicht unmittelbar mit OER befasst sind, beispielsweise über Twitter, Newsletter sowie die Webseiten der OERTransferpartner. Die Transferpartner befinden sich entweder an Universitäten oder Forschungseinrichtungen und unterstützen OERinfo, um OER in die verschiedenen Bildungsbereiche zu tragen. In diesem Kontext erfolgen regelmässig Massnahmen im Bereich OER, wie die Teilnahme an Barcamps und Konferenzen. Ausserdem führen sie eigene Workshops und Schulungen auf internen und externen Veranstaltungen durch. Diese Veranstaltungen wurden genutzt, um den Fragebogen zu verteilen, der online über einen Link oder einen QR-Code bereitgestellt wurde. Der Start der Umfrage erfolgte ab Anfang Februar 2019, für die folgende Analyse wurden Daten bis Ende Oktober 2019 einbezogen.

\section{Ergebnisdiskussion}

Der Erhebungszeitraum für die Umfrage betrug neun Monate. Insgesamt wurde der Link zur Umfrage 333 Mal angeklickt, dabei kam es zu 201 vollständigen und 131 unvollständigen Antworten beziehungsweise Teilantworten. Der auf dieser Basis bereinigte Datenkorpus umfasste 201 Voll- und sechs Teilantworten $(n=207)$.

Die Verteilung der Umfrageteilnehmenden zeigt, dass die meisten von ihnen an OER-bezogenen Veranstaltungen teilgenommen haben. Nur 52 haben von anderen Quellen (Newsletter, Partner-Websites oder Sonstiges) über die Umfrage erfahren. Es ist jedoch wahrscheinlich, dass Letztere zumindest den Begriff OER kannten, da im Einladungstext zu der Umfrage auf OER hingewiesen wurde. Ex ante zur folgenden 
Darstellung der Ergebnisse muss somit berücksichtigen werden, dass es sich um eine Positivselektion handelt, da die Teilnehmenden überwiegend im Rahmen von OERMassnahmen an der Umfrage teilnahmen.

\begin{tabular}{|l|l|l|l|l|}
\hline \multicolumn{2}{|l|}{$\begin{array}{l}\text { Wie sind Sie auf diese Umfrage aufmerksam } \\
\text { geworden? }\end{array}$} & Häufigkeit & Prozent & $\begin{array}{c}\text { Gültiger Pro- } \\
\text { zentsatz }\end{array}$ \\
\hline Gültig & Newsletter & 17 & 8,2 & 8,5 \\
\hline & OER Vorträge & 33 & 15,9 & 16,4 \\
\hline & OER Webinar & 7 & 3,4 & 3,5 \\
\hline & OER Seminar & 49 & 23,7 & 24,4 \\
\hline & OERcamp & 29 & 14,0 & 14,4 \\
\hline & OERinfo Webseite & 16 & 7,7 & 8,0 \\
\hline & Partner Webseite & 18 & 8,7 & 9,0 \\
\hline & Sonstiges & 11 & 5,3 & 5,5 \\
\hline & Insgesamt & 201 & 97,1 & 100 \\
\hline Insgesamt & Fehlend & 6 & 2,9 & \\
\hline
\end{tabular}

Tab. 2.: Verteilung der Teilnehmenden.

Bezüglich des Bildungsbereichs stammen die meisten Teilnehmenden aus dem Bereich Hochschule ( $n=114)$, gefolgt von der Weiterbildung $(n=44)$, der Schule $(n=31)$ und einem geringen Anteil aus der beruflichen Bildung $(n=13)$. Die geringen Zahlen für die Schule sind überraschend, da die Befunde in der Literatur darauf hindeuten, dass OER vor allem im schulischen Bereich verbreitet ist (Deutscher Bildungsserver 2016; Richter und Ehlers 2012). Davon abgesehen sind die weiteren Zahlen im Einklang mit der geringen Verbreitung von OER in der beruflichen Bildung.

\begin{tabular}{|l|l|l|l|l|l|}
\hline \multicolumn{2}{|l|}{ Zu welchem Bildungsbereich gehören Sie? } & Häufigkeit & Prozent & $\begin{array}{l}\text { Gültiger } \\
\text { Prozent- } \\
\text { satz }\end{array}$ & $\begin{array}{l}\text { Kumulier- } \\
\text { ter Pro- } \\
\text { zentsatz }\end{array}$ \\
\hline Gültig & Schule & 31 & 15,0 & 15,3 & 15,3 \\
\hline & Hochschule & 114 & 55,1 & 56,4 & 71,8 \\
\hline & Berufliche Bildung & 13 & 6,3 & 6,4 & 78,2 \\
\hline & Erwachsenen-/ Weiterbildung & 44 & 21,3 & 21,8 & 100,0 \\
\hline & Insgesamt & 202 & 97,6 & 100,0 & \\
\hline Fehlend & & 5 & 2,4 & & \\
\hline Insgesamt & & 207 & 100 & & \\
\hline
\end{tabular}

Tab. 3.: Bildungsbereich. 
Hinsichtlich der Frage nach den Faktoren Gefühlen und Emotionen ist besonders der erste Abschnitt interessant (Tabelle 4). Hier wurde, ohne OER explizit zu nennen, nach den für OER als elementar verstandenen Konzepten und Werten wie der Idee des Teilens, Austausches und der Zusammenarbeit gefragt. Die Zustimmung zu allen diesen Kategorien kann als sehr hoch eingeordnet werden und deutet auf die Wichtigkeit dieser für die Befragten hin. Besonders die prinzipielle Idee des Austausches und der Zusammenarbeit erhält mit 4.66 die höchste Zustimmung, gefolgt von Teilen als zentralem Bestandteil von Bildung.

\begin{tabular}{|c|c|c|c|c|c|c|}
\hline \multicolumn{2}{|c|}{$\begin{array}{l}\text { Inwiefern stimmen Sie } \\
\text { folgenden Aussagen } \\
\text { zu? (1-5) }\end{array}$} & \multirow{2}{*}{$\begin{array}{l}\text { [Den Aus- } \\
\text { tausch und } \\
\text { die Zusam- } \\
\text { menarbeit mit } \\
\text { Kolleg*innen } \\
\text { empfinde ich } \\
\text { als wichtig.] }\end{array}$} & \multirow{2}{*}{$\begin{array}{l}\text { [Das Teilen } \\
\text { hilft mir, } \\
\text { Feedback von } \\
\text { Anderen zu } \\
\text { erhalten.] } \\
\\
205\end{array}$} & \multirow{2}{*}{$\begin{array}{l}\text { [Es macht mir } \\
\text { Freude, wenn } \\
\text { andere meine } \\
\text { Materialien } \\
\text { verwenden.] }\end{array}$} & \multirow{2}{*}{$\begin{array}{l}\text { [Meine Materi- } \\
\text { alien zu teilen, } \\
\text { hilft bei der } \\
\text { Verbreitung } \\
\text { meiner Ideen.] } \\
202 \\
\end{array}$} & \multirow{2}{*}{$\begin{array}{l}\text { [Teilen ist ein } \\
\text { zentraler Be- } \\
\text { standteil von } \\
\text { Bildung.] } \\
\\
204 \\
\end{array}$} \\
\hline \multirow{2}{*}{$\mathrm{N}$} & Gültig & & & & & \\
\hline & Fehlend & 2 & 2 & 4 & 5 & 3 \\
\hline $\begin{array}{l}\text { Mittel- } \\
\text { wert }\end{array}$ & & 4,66 & 4,68 & 4,19 & 4,24 & 4,03 \\
\hline Median & & 5,00 & 5,00 & 4,00 & 4,00 & 4,00 \\
\hline SD & & ,739 & ,715 & ,879 & ,888 & ,959 \\
\hline
\end{tabular}

Tab. 4.: $\quad$ Einstellungen zum Teilen, Austauschen und zur Zusammenarbeit.

Tabelle 5 zeigt den von den Teilnehmern angegebenen Kenntnisstand über OER (Mittelwert 3,78). 33,9\% gaben ein moderates Niveau an, 28,6 \% ein hohes. Diese Zahlen lassen sich primär dadurch erklären, dass das Wissen vor allem in den OERWorkshops und OERcamps mit vielen Anfängern eher moderat ausfällt. Nur 6,3\% gaben an, kein Wissen über OER zu haben, was die Annahme weiter verstärkt, dass nur diejenigen, die mit OER vertraut sind, an der Umfrage teilgenommen haben.

\begin{tabular}{|c|c|c|}
\hline \multicolumn{3}{|c|}{$\begin{array}{l}\text { Wie schätzen Sie lhre Kenntnisse zu OER ein? } \\
\text { (1 keine - } 6 \text { sehr hoch) }\end{array}$} \\
\hline \multirow[t]{2}{*}{ N } & Gültig & 189 \\
\hline & Fehlend & 18 \\
\hline Mittelwert & 3,78 & \\
\hline Median & 4,00 & \\
\hline Standardabweichung & 1,280 & \\
\hline
\end{tabular}

Tab. 5.: OER-Kenntnisse. 
Abgeleitet aus der breiteren Diskussion in der OER-Literatur (Delgado, Delgado, und Hilton 2019; Pawlowski und Bick 2012; Wiley und Hilton 2018), sollten die Teilnehmenden im folgenden Abschnitt ihre Zustimmung zu dem häufig postulierten Mehrwert von OER angeben. Während die Ergebnisse eine hohe Zustimmung für alle Punkte zeigen, sind abstrakte Konzepte wie Teilen (4.29) und Austausch (4.25) besonders hoch, während die Zustimmung leicht abnimmt, wenn konkrete Vorteile genannt werden, beispielsweise die Rechtssicherheit (3.76). Diese Ergebnisse stimmen mit anderen Studien überein, die besagen, dass die Rechtsunsicherheit eine der wesentlichen Hürden für den Einsatz von OER ist (Bozkurt, Koseoglu, und Singh 2019).

\begin{tabular}{|c|c|c|c|c|c|}
\hline \multirow{2}{*}{$\begin{array}{l}\text { Inwiefern stimmen Sie folgenden Aussa- } \\
\text { gen zum Mehrwert von OER zu? (1-5) }\end{array}$} & \multicolumn{2}{|c|}{$\mathrm{N}$} & \multirow{2}{*}{$\begin{array}{l}\text { Mittel- } \\
\text { wert }\end{array}$} & \multirow{2}{*}{ Median } & \multirow{2}{*}{ SD } \\
\hline & Gültig & Fehlend & & & \\
\hline $\begin{array}{l}\text { [OER ermöglichen eine Kultur des Tei- } \\
\text { lens.] }\end{array}$ & 198 & 9 & 4,29 & 4,00 & ,839 \\
\hline [OER erleichtern den Wissensaustausch.] & 197 & 10 & 4,25 & 4,00 & ,873 \\
\hline $\begin{array}{l}\text { [OER fördern das innovative Lehren und } \\
\text { Lernen.] }\end{array}$ & 197 & 10 & 3,93 & 4,00 & ,953 \\
\hline $\begin{array}{l}\text { [OER erleichtern die Vorbereitung der } \\
\text { eigenen Lehre.] }\end{array}$ & 196 & 11 & 3,93 & 4,00 & ,984 \\
\hline $\begin{array}{l}\text { [OER erleichtern die Aktualisierung von } \\
\text { Materialien.] }\end{array}$ & 198 & 9 & 3,96 & 4,00 & ,934 \\
\hline $\begin{array}{l}\text { [OER ermöglichen einen rechtssicheren } \\
\text { Einsatz von Materialien.] }\end{array}$ & 197 & 10 & 3,76 & 4,00 & ,985, \\
\hline
\end{tabular}

Tab. 6.: OER-Mehrwert.

Bemerkenswert ist, dass eine Analyse einer statistischen Beziehung zwischen den Variablen Kenntnisstand über OER und der Wahrnehmung des Mehrwerts von OER mittels Spearman-Rho nur teilweise eine signifikante Korrelation ergab (Tabelle 7). Die eindeutige Korrelation lässt sich nur für die eher abstrakten Wert wie Teilen und Wissensaustausch beobachten. Dies könnte darauf hindeuten, dass OER bei den Teilnehmenden eine positive Konnotation haben die vergleichsweise unabhängig vom Kenntnisstand über OER ist und auf die Rigidität der Überzeugungen hinweist. 


\begin{tabular}{|c|c|c|c|}
\hline & & & $\begin{array}{l}\text { [Wie schätzen Sie Ihre Kenntnis- } \\
\text { se zu OER ein?] }\end{array}$ \\
\hline & [Wie schätzen Sie Ihre & Korrelationskoeffizient & 1,000 \\
\hline & [Kenntnisse zu OER ein?] & Sig. (2-teilig) & . \\
\hline & & $\mathrm{N}$ & 189 \\
\hline & [OER ermöglichen eine & Korrelationskoeffizient &, $244^{\star \star}$ \\
\hline & & Sig. (2-teilig) &, 001 \\
\hline & & $\mathrm{N}$ & 186 \\
\hline & [OER erleichtern den & Korrelationskoeffizient & $209^{\star \star}$ \\
\hline & & Sig. (2-teilig) &, 004 \\
\hline 욷 & & $\mathrm{N}$ & 185 \\
\hline$\stackrel{n}{n}$ & [OER fördern das innova- & Korrelationskoeffizient &, 070 \\
\hline 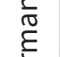 & tive Lehren und Lernen.] & Sig. (2-teilig) & ,342 \\
\hline 离 & & $\mathrm{N}$ & 186 \\
\hline & [OER erleichtern die Vor- & Korrelationskoeffizient &, 105 \\
\hline & bereitung der eigenen & Sig. (2-teilig) &, 157 \\
\hline & & $\mathrm{N}$ & 184 \\
\hline & [OER erleichtern die & Korrelationskoeffizient &, 126 \\
\hline & Aktualisierung von Mate- & Sig. (2-teilig) & 086 \\
\hline & & $\mathrm{N}$ & 186 \\
\hline & [OER ermöglichen einen & Korrelationskoeffizient &, 094 \\
\hline & rechtssicheren Einsatz & Sig. (2-teilig) &, 204 \\
\hline & & $N$ & 185 \\
\hline
\end{tabular}

Tab. 7.: Zusammenhang zwischen OER-Kenntnisse und Nutzen. [Die Korrelation ist signifikant auf der 0,01er Marke (2-teilig)].

Ein weiterer Fokus der Analyse bestand darin, zu untersuchen, ob eine Diskrepanz zwischen Überzeugungen und Gefühlen über OER (kognitive und affektive Komponente) und der tatsächlichen Nutzung oder Absicht (Verhaltenskomponente) existiert. Dafür war der letzte Abschnitt der Umfrage relevant, da hier die Teilnehmenden befragt wurden, ob sie OER bereits genutzt, veröffentlicht oder überarbeitet haben oder ob sie dies zukünftig beabsichtigen.

Tabelle 8 zeigt, dass $102(49,3 \%)$ der Teilnehmenden OER bereits verwendet haben, während $64(30,9 \%)$ sie veröffentlicht und $58(28 \%)$ sie überarbeitet haben. Teilnehmende, die OER nutzen, gehören ebenso zu denjenigen, die OER veröffentlicht und überarbeitet haben. Das erste Engagement in der Nutzung von OER scheint demnach weitere OER-Aktivitäten zu befördern. Zusammenfassend sind über die Hälfte der Teilnehmenden bereits im Bereich OER aktiv. 


\begin{tabular}{|c|c|c|c|c|c|c|}
\hline & \multicolumn{2}{|c|}{$\begin{array}{l}\text { [Ich verwende OER in } \\
\text { meiner Lehre/ meinen } \\
\text { Veranstaltungen.] }\end{array}$} & \multicolumn{2}{|c|}{$\begin{array}{l}\text { [Ich habe bereits eigene } \\
\text { OER veröffentlicht.] }\end{array}$} & \multicolumn{2}{|c|}{$\begin{array}{l}\text { [Ich habe bereits OER } \\
\text { von anderen überarbei- } \\
\text { tet.] }\end{array}$} \\
\hline & Nein & $\mathrm{Ja}$ & Nein & $\mathrm{Ja}$ & Nein & $\mathrm{Ja}$ \\
\hline Anzahl & 92 & 102 & 131 & 64 & 136 & 58 \\
\hline $\begin{array}{l}\text { Gültiger Pro- } \\
\text { zentsatz }\end{array}$ & 44,4 & 49,3 & 63,3 & 30,9 & 65,7 & 28 \\
\hline Fehlend & $11(5,3 \%)$ & & $9(4,3 \%)$ & & $10(4,8 \%)$ & \\
\hline Insgesamt & $207(100 \%)$ & & $207(100 \%)$ & & $207(100 \%)$ & \\
\hline
\end{tabular}

Tab. 8.: Verhalten in Bezug auf OER.

Diese moderate Zahl von OER-Praktikerinnen und -Praktiker ist vermutlich darauf zurückzuführen, dass viele Teilnehmende an OERcamps oder OER-Workshops teilgenommen haben, die sich hauptsächlich an Anfängerinnen und Anfänger richten. Die Teilnehmenden werden hier mit den notwendigen Fähigkeiten vertraut gemacht, um zukünftig OER in ihrer Praxis zu nutzen. Dies zeigt sich in der Absicht von 64 der Umfrageteilnehmenden, OER in zukünftigen Veranstaltungen zu nutzen. Nur 23 Teilnehmende gaben an, keine Absicht bezüglich der Nutzung von OER zu haben. Die Absicht, OER in Zukunft zu veröffentlichen, wurde von 87 Personen markiert, 40 von ihnen verwenden bereits OER, während 31 keine Erfahrung haben. Analoge Ergebnisse finden sich in Bezug auf Pläne zur Überarbeitung von OER.

\begin{tabular}{|c|c|c|c|c|c|c|}
\hline & \multicolumn{2}{|c|}{$\begin{array}{l}\text { [Beabsichtigen Sie zu- } \\
\text { künftig OER in Ihren eige- } \\
\text { nen Veranstaltungen zu } \\
\text { verwenden?] }\end{array}$} & \multicolumn{2}{|c|}{$\begin{array}{l}\text { [Beabsichtigen Sie zu- } \\
\text { künftig eigene OER zu } \\
\text { veröffentlichen?] }\end{array}$} & \multicolumn{2}{|c|}{$\begin{array}{l}\text { [Beabsichtigen Sie zu- } \\
\text { künftig OER von anderen } \\
\text { zu überarbeiten?] }\end{array}$} \\
\hline & Nein & $\mathrm{Ja}$ & Nein & $\mathrm{Ja}$ & Nein & $\mathrm{Ja}$ \\
\hline Anzahl & 23 & 64 & 43 & 87 & 50 & 88 \\
\hline $\begin{array}{l}\text { Gültiger Pro- } \\
\text { zentsatz }\end{array}$ & 26,4 & 73,6 & 33,1 & 66,9 & 36,2 & 63,8 \\
\hline Fehlend & $120(58 \%)$ & & $77(37,2 \%)$ & & $69(33,3 \%)$ & \\
\hline Insgesamt & $207(100 \%)$ & & $207(100 \%)$ & & $207(100 \%)$ & \\
\hline
\end{tabular}

Tab. 9.: Absicht, sich an OER zu beteiligen.

Bemerkenswert ist, dass ein Test von Intention und Verhalten (abhängige Variablen) gegen den Bildungssektor (unabhängige Variable) mit Phi, Cramer's V und dem Kontingenzkoeffizienten keine signifikante Korrelation ergab. 


\begin{tabular}{|l|l|l|l|}
\hline \multicolumn{3}{|l|}{ Symmetrische Massnahmen des Bildungsbereichs und nach Veröffentlichung von OER } \\
\hline \multirow{3}{|l|}{ Nominal by Nominal } & Wert & Ungefähre Signifikanz \\
\cline { 2 - 4 } & Cramer's V &, 126 &, 798 \\
\cline { 2 - 4 } & Kontingenzkoeffizient &, 089 &, 798 \\
\hline N von gültigen Fällen & 172 &, 798 \\
\hline
\end{tabular}

Tab. 10.: Korrelation zwischen dem Bildungsbereich und der Veröffentlichung von OER.

\begin{tabular}{|l|l|l|l|}
\hline \multicolumn{3}{|l}{ Symmetrische Massnahmen des Bildungsbereichs und Absicht zur Veröffentlichung von OER } \\
\hline \multirow{3}{*}{ Nominal by Nominal } & & Wert & Ungefähre Signifikanz \\
\cline { 2 - 4 } & Phi & 084 &, 829 \\
\cline { 2 - 4 } & Cramer's V &, 084 &, 829 \\
\cline { 2 - 4 } & Kontingenzkoeffizient &, 083 &, 829 \\
\hline N von gültigen Fällen & 127 & \\
\hline
\end{tabular}

Tab. 11.: Korrelation zwischen dem Bildungsbereich und der Absicht, OER zu veröffentlichen.

Gleichwohl offenbart der Chi-Square Tests (Tabelle 12) eine signifikante Korrelation zwischen dem Kenntnisstand über OER und den Erfahrungen mit der Veröffentlichung von OER. Ähnliche Ergebnisse zeigen sich für Erfahrungen mit der Verwendung und Überarbeitung von OER.

\begin{tabular}{|c|c|c|c|}
\hline \multicolumn{4}{|l|}{ Chi-Quadrat-Tests } \\
\hline & Wert & df & $\begin{array}{l}\text { Asymptotische Sig- } \\
\text { nifikanz (2-seitig) }\end{array}$ \\
\hline Pearson Chi-Quadrat & $67,368^{1}$ & 10 &, 000 \\
\hline Wahrscheinlichkeits-Verhältnis & 71,260 & 10 &, 000 \\
\hline Linear-by-Linear Zuordnung & 28,684 & 1 &, 000 \\
\hline N von gültigen Fällen & 186 & & \\
\hline
\end{tabular}

Tab. 12.: Zusammenhang zwischen den OER-Kenntnissen und der Veröffentlichung von OER.

Es ist beachtenswert, dass sich kein solcher Zusammenhang für den Kenntnisstand und die Absicht OER zu verwenden, zu veröffentlichen oder zu überarbeiten zeigt (exemplarisch dargestellt in Tabelle 13). Da jedoch für beide Korrelationen, Verhalten und Absicht teilweise eine unzureichende Datenmenge existiert, haben diese eine begrenzte Gültigkeit und damit eine eingeschränkte Aussagekraft.

18 Zellen (44,4\%) haben eine Anzahl von weniger als 5 erwartet. Die minimale erwartete Anzahl beträgt ,13. 


\begin{tabular}{|c|c|c|c|}
\hline \multicolumn{4}{|l|}{ Chi-Quadrat-Tests } \\
\hline & Wert & $d f$ & $\begin{array}{l}\text { Asymptotische Sig- } \\
\text { nifikanz (2-seitig) }\end{array}$ \\
\hline Pearson Chi-Quadrat & $12,135^{2}$ & 5 &, 033 \\
\hline Wahrscheinlichkeits-Verhältnis & 12,015 & 5 & 035 \\
\hline Linear-by-Linear Zuordnung & 8,016 & 1 &, 005 \\
\hline N von gültigen Fällen & 122 & & \\
\hline
\end{tabular}

Tab. 13.: Zusammenhang zwischen den OER-Kenntnissen und der Absicht, OER zu veröffentlichen.

Wenn die Ergebnisse zusammengeführt werden, deutet dies darauf hin, dass die Überzeugungen über OER teilweise unabhängig vom Wissen über OER sind, aber Wissen eine zentrale Voraussetzung für die Nutzung von OER bildet.

Die Befragung zu den Herausforderungen für OER in der Bildung offenbart, dass keine entscheidende Barriere existiert, die vorherrschend ist. Bedeutsam ist, dass von den in der Literatur identifizierten Barrieren nur zwei von knapp mehr als der Hälfte der Teilnehmenden angegeben wurden. Dies verdeutlicht, dass keine dieser Herausforderungen als ein elementares Hindernis für das Engagement bezüglich OER wahrgenommen wird. Besonders die mangelnde Akzeptanz von OER scheint für die Teilnehmenden keine wesentliche Herausforderung darzustellen (35,7 Prozent).

\begin{tabular}{|l|l|l|l|l|l|l|}
\hline \multicolumn{6}{|c|}{ Welche Herausforderungen sehen Sie für OER in Ihrem Bildungsbereich? } \\
\hline \multicolumn{2}{|c|}{} & Fehlende Zeit & $\begin{array}{c}\text { Rechtliche } \\
\text { Unsicherheit }\end{array}$ & $\begin{array}{c}\text { Technische } \\
\text { Hürden }\end{array}$ & $\begin{array}{c}\text { Finden von } \\
\text { Material }\end{array}$ & $\begin{array}{c}\text { Mangelnde } \\
\text { Akzeptanz }\end{array}$ \\
\hline \multirow{2}{*}{ N } & Gültig & 207 & 207 & 207 & 207 & 207 \\
\cline { 2 - 8 } & Fehlend & 25 & 22 & 29 & 29 & 29 \\
\hline & Ja & $112(54,1 \%)$ & $104(50,2 \%)$ & $76(36,7 \%)$ & $88(43 \%)$ & $74(35,7 \%)$ \\
\hline
\end{tabular}

Tab. 14.: Herausforderungen für OER.

\section{Implikationen und Grenzen der Ergebnisse}

Ziel des Beitrages war eine systematische und theoretisch gestützte Annäherung an die Rolle von Einstellungen für die Nutzung und Verbreitung von OER in Deutschland. Basierend auf einem Konzept aus der Einstellungsforschung und einem umfragebasierten explorativen Ansatz wurde versucht, die determinierenden Einstellungskomponenten bildungsbereichsübergreifend abzubilden.

23 Zellen (25\%) haben eine Anzahl von weniger als 5 erwartet. Die minimal erwartete Anzahl ist ,32. 
Zusammenfassend lässt sich festhalten, dass unter den Umfrageteilnehmenden, die weitestgehend Teil der breiteren OER-Gemeinschaft sind, eine affirmative Einstellung bezüglich OER in allen drei Einstellungskomponenten vorliegt. Besonders in der affektiven Komponente manifestieren sich die affirmativen Einstellungen in den hohen Zustimmungen zu den mit OER assoziierten Werten und Ideen. Während das Wissen über OER eher moderat ist, sind die Überzeugungen über die mit OER verbundenen Mehrwerte eindeutig hoch. Bemerkenswert ist, dass diese Überzeugungen weitestgehend unabhängig vom Wissen über OER sind. Interessanterweise lässt sich eine Korrelation zwischen Wissen und Überzeugungen lediglich für die eher abstrakten Werte wie Teilen und Austausch herstellen, nicht jedoch, wie möglicherweise vermutet werden könnte, für die konkreten Vorteile wie innovatives Lernen oder Rechtssicherheit. Somit werden die in der Literatur postulierten Mehrwerte, die mit dem Einsatz von OER verbunden sind, zwar von den Teilnehmenden grundsätzlich bestätigt, allerdings haben die genannten greifbaren Vorteile eine geringere Zustimmung als die eher abstrakten. Weiterhin erwähnenswert ist, dass die Ergebnisse keine wesentlichen Herausforderungen aufzeigen, welche die Teilnehmenden als Hindernis empfinden, um OER in ihrer Bildungspraxis zu nutzen. Diese werden zwar genannt, scheinen aber innerhalb der OER-Gemeinschaft eine geringere Relevanz zu besitzen, als häufig in der Literatur angenommen. Die Homogenität dieser OER-Gemeinschaft wird auch dadurch evident, dass keine signifikanten Unterschiede bezüglich der Bildungsbereiche festgestellt werden konnten. Dies ist gerade für den kompetitiven und teilweise honorarbasierten Bildungsbereich der Erwachsenenbildung beachtlich, da besonders hier die Verbreitung von OER gering und besonders dessen Nutzung mit hohen Widerständen verbunden ist (Otto 2019).

Als letzter Aspekt sind für die Handlungskomponente die positiven Gefühle und Emotionen hervorzuheben, die von den Teilnehmenden insgesamt mit der höchsten Zustimmung bedacht werden. Dies ist von immanenter Bedeutung, da hohe Werte in der affektiven Komponente als Vorabbedingung für positives Verhalten angesehen werden (Rosenberg und Hovland 1960; Shrigley 1990). Dieser Zusammenhang zeigt sich auch für den Fall von OER, da hier 166 der 207 Teilnehmenden entweder OER nutzen (102) oder dies beabsichtigen (64). Darauf aufbauend lässt sich vermuten, dass für den Fall von OER affektive und Verhaltenskomponente miteinander verbunden sind. Bei der kognitiven Komponente existiert ein positiver Zusammenhang zwischen der Höhe des Wissens über OER und den praktischen Erfahrungen mit diesen. 


\begin{tabular}{|l|l|l|}
\hline Einstellung & Indikator & Zustimmung \\
\hline Kognitive Komponente & $\begin{array}{l}\text { Moderates Wissen und starke positive Über- } \\
\text { zeugungen über OER }\end{array}$ & Moderat bis Hoch \\
\hline Affektive Komponente & $\begin{array}{l}\text { Affirmative Gefühle und Emotionen für die } \\
\text { hinter OER stehenden Werte und Ideen }\end{array}$ & Sehr Hoch \\
\hline Verhaltenskomponente & $\begin{array}{l}\text { Über Zweidrittel nutzen OER oder beach- } \\
\text { sichtigen dies }\end{array}$ & Sehr Hoch \\
\hline
\end{tabular}

Tab. 15.: Ausprägung der Einstellungskomponenten bezüglich OER.

Abschliessend muss festgehalten werden, dass die gewonnenen Erkenntnisse durch die verwendete Methodik eingeschränkt sind und damit letztlich auch die Aussagekraft in einigen Aspekten limitiert ist. Die Operationalisierung der Komponenten des Einstellungskonzepts ist sehr basal und hätte weiter ausdifferenziert werden können. Zentrale Konzepte wie beispielsweise das Teilen haben in der OER-Gemeinschaft vermutlich sehr wahrscheinlich ein anderes und viel weitreichenderes Verständnis als ausserhalb. OER sind daher mit anderen gesellschaftspolitischen Forderungen verknüpft, die über das tradierte Verständnis von Bildungseinrichtungen und ihrem Leistungsprinzip hinausgehen (Bock und Tribukait 2019). Die Verknappung bei der Operationalisierung der verschiedenen Indikatoren wurde gegen die erwartete Steigerung bei der Rücklaufquote abgewogen und entschieden. Als methodisch schwierig erweist sich weiterhin die Verhaltenskomponente, die ausschliesslich auf die Selbsteinschätzung der Teilnehmenden rekurriert. Eine Verifizierung im Sinne einer Beobachtungsstudie oder Ähnlichem zur Überprüfung der Transformation von Intention in soziale Praktiken ist daher nicht möglich. Darüber hinaus wäre für die Validierung der hohen Werte für die affektive Komponente eine Kontrollgruppe wünschenswert. Dies verweist auf die geringe Rücklaufquote der Umfrage bei Personen, die nicht Teil der OER-Gemeinschaft sind und eventuell als eine Art von Kontrollgruppe hätten fungieren können. Diese quasi Inexistenz ist bemerkenswert, da die Umfrage in vielen für die Bildungsbereiche relevanten Newslettern und Websites, die nicht OER-bezogen sind, verbreitet wurde. Vor dem Hintergrund der Ergebnisse in der Literatur (Deimann, Neumann, und Muuß-Merholz 2015; Orr, Neumann, und Muuß-Merholz 2017; Surmann und Echterhoff 2018) kann dies somit als konfirmatorisch für den häufig geäusserten, aber bisher empirisch nicht belegten Verdacht einer geringen Verbreitung und Nutzung von OER ausserhalb eines engagierten Zirkels sowie die Existenz einer engagierten OER-Gemeinschaft gedeutet werden. 


\section{Zusammenfassung und Ausblick}

In einer Gesamtbetrachtung, so zeigen die Ergebnisse, sind die Einstellungen nicht das zentrale Hindernis für die Nutzung von OER in Deutschland, vielmehr scheint es die mangelnde Verbreitung von OER zu sein. Bezüglich der Verbreitung lässt sich konstatieren, dass sowohl Intention als auch Verhalten nur innerhalb einer OERGemeinschaft existieren, die allerdings starke Emotionen und Überzeugungen mit den Kernideen und Mehrwerten von OER verbindet. Diesem Argument folgend wäre der häufig geforderte Abbau struktureller Barrieren ein notwendiger, aber keineswegs hinreichender Grund, um die Nutzung und Verbreitung von OER zu erhöhen. Die Ergebnisse zeigen nicht überraschend, dass primär die affektive Komponente ein Prädiktor sowohl für die Absicht als auch die Nutzung von OER ist. Die Nutzung steht wiederum in Abhängigkeit zur Höhe des Wissensstandes über OER. Folgerichtig wäre daher für die weitere Verbreitung von OER die Einführung von permanenten und systematischen Fortbildungsprogrammen in Bildungseinrichtungen, bestenfalls verpflichtend oder zumindest in irgendeiner Form goutiert. Auf einer grundlegenderen Ebene muss dann allerdings ex-ante sowohl auf Akteurs- als auch auf Strukturebene der Grundgedanke des Teilens, Austausches und der Zusammenarbeit im Kontext des Lehrens und Lernens in Bildungseinrichtungen, unabhängig von OER, stärker verankert werden. Obwohl diese Grundgedanken über OER hinaus Geltung besitzen, scheint besonders die teilweise subversive Affirmation dieser in der OER-Bewegung problematisch. Eine Möglichkeit wäre daher der Versuch, auf diskursiver Ebene iterativ zu bestimmen, welche Ideen und ebenso Handlungsimplikationen an diese recht abstrakten Begriffe in einer Bildungseinrichtung anschlussfähig sind und mit diesen verknüpft werden können. Neben einem Gemeinschaftsprinzip koexistiert und konkurriert dieses in Bildungseinrichtungen stets mit einem Leistungs- und damit in finaler Instanz ebenso mit einem Selektionsprinzip, das auch durch (ein Mehr von) OER, Open Education und Openness nicht erodiert werden kann.

Letztlich sind es somit aber gerade die den OER inhärenten, aber häufig nicht explizit genug in Erscheinung tretenden (medien-)pädagogischen (Mehr-)Werte und Ideen, die über das Kernkonzept und einer teilweise normativen Ausrichtung bis hin zu einer Openness-Bewegung hinaus Anziehungskraft und Wirkmächtigkeit besitzen. Eine Herausforderung könnte es daher sein, diese zukünftig ostentativer herauszustellen und nicht innerhalb einer OER-Gemeinschaft zu verbergen. Insofern kann hier Kerres (2019) und anderen nur sekundiert werden, nicht mit einer normativen Zielsetzung zu missionieren, sondern OER als eine (Teil-)Antwort auf eine breitere Herausforderung des Lehrens und Lernens in der digitalen Welt zu begreifen, zu vermitteln und bildungsbereichsübergreifend zu integrieren. 


\section{Literatur}

Allen, Chris T, Karen A Machleit, und Susan Schultz Kleine. 1992. «A Comparison of Attitudes and Emotions as Predictors of Behavior at Diverse Levels of Behavioral Experience». Journal of Consumer Research 18 (4): 493-504.

Allport, Gordon. 1935. «Attitudes». In Handbook of Social Psychology, herausgegeben von C. Murchison und MA Worcester, 789-844. Clark University Press.

Avey, James B., Tara S. Wernsing, und Fred Luthans. 2008. «Can Positive Employees Help Positive Organizational Change? Impact of Psychological Capital and Emotions on Relevant Attitudes and Behaviors». The Journal of Applied Behavioral Science 44 (1): 48-70. https:// doi.org/10.1177/0021886307311470.

Bayne, Siân, Jeremy Knox, und Jen Ross. 2015. «Open education: the need for a critical approach». Learning, Media and Technology 40 (3): 247-50. https://doi.org/10.1080/1743988 4.2015.1065272.

Bellinger, Franziska, und Kerstin Mayrberger. 2019. "Systematic Literature Review zu Open Educational Practices (OEP) in der Hochschule im europäischen Forschungskontext». MedienPädagogik: Zeitschrift für Theorie und Praxis der Medienbildung 34 (Research and Open Educational Resources - A snapshot on Europe). https://doi.org/10.21240/ mpaed/34/2019.02.18.X.

Bock, Annekatrin, und Maren Tribukait. 2019. «Kultur des Teilens». MedienPädagogik: Zeitschrift für Theorie und Praxis der Medienbildung 34 (Research and Open Educational Resources - A snapshot on Europe). https://doi.org/10.21240/mpaed/34/2019.02.22.X.

Bohner, Gerd, und Nina Dickel. 2010. «Attitudes and Attitude Change». Annual Review of Psychology 62 (1): 391-417. https://doi.org/10.1146/annurev.psych.121208.131609.

Bozkurt, Aras, Suzan Koseoglu, und Lenandlar Singh. 2019. «An analysis of peer reviewed publications on openness in education in half a century: Trends and patterns in the open hemisphere». Australasian Journal of Educational Technology 35 (4): 68-97. https://doi. $\operatorname{org} / 10.14742$ /ajet.4252.

Breidenstein, Georg. 2009. «Allgemeine Didaktik und praxeologische Unterrichtsforschung BT - Perspektiven der Didaktik: Zeitschrift für Erziehungswissenschaft». In , herausgegeben von Meinert A Meyer, Manfred Prenzel, und Stephanie Hellekamps, 201-15. Wiesbaden: VS Verlag für Sozialwissenschaften. https://doi.org/10.1007/978-3-531-91775-7_14.

Chaiklin, Harris. 2011. «Attitudes, Behavior, and Social Practice». The Journal of Sociology \& Social Welfare 38 (1): 31-54.

Deimann, Markus. 2018. «Lernen mit Open Educational Resources». In Lernen mit Bildungstechnologien: Praxisorientiertes Handbuch zum intelligenten Umgang mit digitalen Medien, herausgegeben von Helmut Niegemann und Armin Weinberger, 1-10. Berlin, Heidelberg: Springer Berlin Heidelberg. https://doi.org/10.1007/978-3-662-54373-3_58-1.

Deimann, Markus. 2019. «Forschung und Open Educational Resources - Eine Momentaufnahme für Europa». MedienPädagogik: Zeitschrift für Theorie und Praxis der Medienbildung 34 (Research and Open Educational Resources - A snapshot on Europe). https://doi.org/https://doi.org/10.21240/mpaed/34.X. 
Deimann, Markus, Jan Neumann, und Jöran Muuß-Merholz. 2015. «Whitepaper. Open Educational Resources (OER) an Hochschulen in Deutschland - Bestandsaufnahme und Potenziale 2015». Hagen, Hamburg und Köln.

Delgado, Huimei, Michael S. Delgado, und John Hilton. 2019. «On the efficacy of open educational resources: Parametric and nonparametric analyses of a university calculus class». International Review of Research in Open and Distance Learning 20 (1): 185-203.

Deutscher Bildungsserver. 2016. «Machbarkeitsstudie zum Aufbau und Betrieb von OERInfrastrukturen in der Bildung». Frankfurt am Main. 2016. http://nbn-resolving.org/ urn:nbn:de:0111-pedocs-117154.

Dillman, Don A, Michael D Sinclair, und Jon R Clark. 1993. "Effects of Questionnaire Length, Respondent-Friendly Design, and a Difficult Question on Response Rates for OccupantAddressed Census Mail Surveys». The Public Opinion Quarterly 57 (3): 289-304.

Fecher, Benedikt, und Sascha Friesike. 2014. «Open Science: One Term, Five Schools of Thought». In Opening Science: The Evolving Guide on How the Internet is Changing Research, Collaboration and Scholarly Publishing, herausgegeben von Sönke Bartling und Sascha Friesike, 17-47. Cham: Springer International Publishing. https://doi.org/10.1007/978-3319-00026-8_2.

Flam, Helena, und Debra King. 2005. Emotions and social movements. Emotions and Social Movements. London and New York: Routledge. https://doi.org/10.4324/9780203013526.

Gawronski, Bertram, Wilhelm Hofmann, und Christopher J. Wilbur. 2006. «Are ,implicit' attitudes unconscious?» Consciousness and Cognition 15 (3): 485-99. https://doi.org/10.1016/j. concog.2005.11.007.

Giddens, Anthony. 1979. "Central Problems in Social Theory». Central Problems in Social Theory. https://doi.org/10.1007/978-1-349-16161-4.

Gourlay, Lesley. 2015. «Open education as a 'heterotopia of desire'». Learning, Media and Technology 40 (3): 310-27. https://doi.org/10.1080/17439884.2015.1029941.

Grimm, Pamela. 2010. «Pretesting a Questionnaire». In Wiley International Encyclopedia of Marketing. Chichester, UK: John Wiley \& Sons, Ltd. https://doi.org/10.1002/9781444316568. wiem02051.

Guo, Yuqing, Muhua Zhang, Curtis J. Bonk, und Yan Li. 2015. «Chinese faculty members' Open Educational Resources (OER) usage status and the barriers to OER development and usage». International Journal of Emerging Technologies in Learning 10 (5): 59-65. https://doi. org/10.3991/ijet.v10i5.4819.

Guyer, Joshua J., und Leandre R. Fabrigar. 2015. «Attitudes and Behavior». In International Encyclopedia of the Social \& Behavioral Sciences: Second Edition, 183-89. https://doi. org/10.1016/B978-0-08-097086-8.24007-5.

Jasper, James M. 2011. «Emotions and Social Movements: Twenty Years of Theory and Research». Annual Review of Sociology 37 (1): 285-303. https://doi.org/10.1146/annurevsoc-081309-150015. 
Jepson, Christopher, David A. Asch, John C. Hershey, und Peter A. Ubel. 2005. «In a mailed physician survey, questionnaire length had a threshold effect on response rate». Journal of Clinical Epidemiology 58 (1): 103-5. https://doi.org/10.1016/j.jclinepi.2004.06.004.

Kanwar, Asha., und S Uvalić-Trumbić. 2011. A basic guide to open educational resources (OER). UNESCO and Commonwealth of Learning. Commonwealth of Learning. https://doi. org/10.1111/j.1467-8535.2012.01378.x.

Kerres, Michael. 2019. "Offene Bildungsressourcen und Open Education: Openness als Bewegung oder als Gefüge von Initiativen?» MedienPädagogik: Zeitschrift für Theorie und Praxis der Medienbildung 34 (Research and Open Educational Resources - A snapshot on Europe). https://doi.org/10.21240/mpaed/34/2019.02.17.X.

Kerres, Michael, Tobias Hölterhof, Gianna Scharnberg, und Nadine Schröder. 2019. «EduArc. Eine Infrastruktur zur hochschulübergreifenden Nachnutzung digitaler Lernmaterialien.» Synergie. Fachmagazin für Digitalisierung in der Lehre \#07.

Leandre R. Fabrigar, Tara K. MacDonald, Duane T. Wegener. 2005. «The Matrix of Attitude-Relevant Influences». In The Handbook of Attitudes, 79-124. Routledge Handbooks Online. https://doi.org/10.4324/9781410612823.ch3.

Lechtenbörger, Jens. 2019. «Erstellung und Weiterentwicklung von Open Educational Resources im Selbstversuch». MedienPädagogik: Zeitschrift für Theorie und Praxis der Medienbildung 34 (Research and Open Educational Resources - A snapshot on Europe). https://doi. org/10.21240/mpaed/34/2019.03.02.X.

Mayrberger, Kerstin. 2018. «Dokumentation der OER-Info Projekte 2017/2018». Synergie. Fachmagazin für Digitalisierung in der Lehre Sonderband: 284. https://doi. org/10.25592/978.3.924330.65.1.

Meinefeld, Werner. 1977. Einstellung und soziales Handeln. rororo-Studium: Sozialwissenschaft. Rowohlt.

OPAL. 2011. «Beyond OER: Shifting focus to Open Educational Practices». https://duepublico. uni-duisburg-essen.de/servlets/DerivateServlet/Derivate-25907/OPALReport2011_Beyond_OER.pdf.

Orr, Dominic, Jan Neumann, und Joeran Muuß-Merholz. 2017. «German OER Practices and Policy - from Bottom-up to Top-down Initiatives». Moskau.

Otto, Daniel. 2019. «Adoption and Diffusion of Open Educational Resources (OER) in Education». The International Review of Research in Open and Distributed Learning 20 (5 SE-Research Articles): 122-40. https://doi.org/10.19173/irrodl.v20i5.4472.

Pawlowski, Jan M., und Markus Bick. 2012. "Open educational resources». Business and Information Systems Engineering 4 (4): 209-12. https://doi.org/10.1080/15228959.2015.109567 1.

Percy, Tanya, und Jean Paul Van Belle. 2012. «Exploring the barriers and enablers to the use of open educational resources by university academics in Africa». In IFIP Advances in Information and Communication Technology, herausgegeben von Imed Hammouda, Björn Lundell, Tommi Mikkonen, und Walt Scacchi, 378 AICT:112-28. Berlin, Heidelberg: Springer Berlin Heidelberg. https://doi.org/10.1007/978-3-642-33442-9_8. 
Peters, Michael A. 2008. «The History and Emergent Paradigm of Open Education». In Open Education and Education for Openness, 3-15.

Peters, Michael A., und Peter Roberts. 2011. «Introduction The Virtues of Openness». In Virtues of Openness | Education, Science, and Scholarship in the Digital Age, 1-16. New York: Routledge.

Richter, Thomas, und Ulf Daniel Ehlers. 2012. «Barriers and Motivators for Using Open Educational Resources in Schools». Open Ed 2010: The Seventh Annual Open Education Conference, Nr. 2010 (September): 413-24. http://openaccess.uoc.edu/webapps/o2/bitstream/10609/4868/6/Richter_editat2.pdf.

Rosenberg, Milton J., und Carl I. Hovland. 1960. «Cognitive, affective, and behavioral components of attitudes». In Attitude Organization and Change: An Analysis of Consistency among Attitude Components, herausgegeben von Milton J. Rosenberg und C.I. Hovland, Yale studi:1-14. New Haven: Yale University Press.

Scheve, Christian von. 2011. "Die soziale Konstitution und Funktion von Emotion: Akteur, Gruppe, normative Ordnung». Zeitschrift für Erziehungswissenschaft 14 (2): 207-22. https:// doi.org/10.1007/s11618-011-0206-3.

Schmid, Ulrich, Lutz Goertz, und Julia Behrens. 2017. «Digitales Lernen an Schulen». Monitor Digitale Bildung. 2017. https://doi.org/10.11586/2017014.

Schmid, Ulrich, Goertz Lutz, Sabine Radomski, und Julia Behrens. 2017. «Die Hochschulen im digitalen Zeitalter». Monitor Digitale Bildung. https://doi.org/10.11586/2017014.

Shrigley, R L. 1990. «Attitude and Behaviour are Correlates». Journal of Research in Science Teaching 27 (2): 97-113.

Stark, Lisa, Babette Park, und Roland Brünken. 2018. «Emotionen beim Lernen mit Multimedia». In Digitalisierung und Bildung, herausgegeben von Silke Ladel, Julia Knopf, und Armin Weinberger, 141-58. Wiesbaden: Springer Fachmedien Wiesbaden. https://doi. org/10.1007/978-3-658-18333-2_8.

Surmann, Caroline, und Charlotte Echterhoff. 2018. «OERinfo - Die Richtlinie zur Förderung von offenen Bildungsmaterialien». In Projekte der BMBF-Förderung OERinfo 2017/2018. Sonderband zum Fachmagazin Synergie, 10-17.

UNESCO. 2002. "Forum on the Impact of Open Courseware for Higher Education in Developing Countries Final report UNESCO». Paris, 1-3 July 2002.

UNESCO. 2019. Guidelines on the Development of Open EducationalResources Policies. UNESCO, and Burnaby, BC, Canada: Commonwealth of Learning,.

Veresova, Marcela, und Dana Mala. 2016. «Attitude toward School and Learning and Academic Achievement of Adolescents». In ICEEPSY 2016: 7th International Conference on Education and Educational Psychology, 870-76. https://doi.org/10.15405/epsbs.2016.11.90.

Weede, Erich. 1992. Mensch und Gesellschaft: Soziologie aus der Perspektive des methodologischen Individualismus. Tübingen: Mohr Siebeck Gmbh \& Co. K.

Wiley, David, und John Hilton. 2018. «Defining OER-enabled pedagogy». International Review of Research in Open and Distance Learning 19 (4): 133-47. https://doi.org/10.19173/irrodl. v19i4.3601. 